\title{
A Simple Deterministic Model for the Spread of Hand, Foot and Mouth Disease (HFMD) in Sarawak
}

\author{
Felix Chuo Sing Tiing, \\ Department of Computational Science and \\ Mathematics, \\ Universiti Malaysia Sarawak \\ felixchuo@gmail.com
}

\author{
Jane Labadin \\ Department of Computational Science and \\ Mathematics, \\ Universiti Malaysia Sarawak \\ ljane@fit.unimas.my
}

\begin{abstract}
Hand, foot and mouth disease (HFMD) is considered a common disease among children. However, HFMD recent outbreaks in Sarawak had caused many death particularly children below the age of ten. In this study we are building a simple deterministic model based on the SIR (SusceptibleInfected-Recovered) model to predict the number of infected and the duration of an outbreak when it occurs. Our findings show that the disease spread quite rapidly and the parameter that may be able to control that would be the number of susceptible persons. We hope the model will allow public health personnel to plan intervention in an effective manner in order to reduce the effect of the disease in the coming outbreak.
\end{abstract}

\section{Introduction}

Hand, foot and mouth disease (HFMD) is a common illness of infant and children. HFMD is caused by enteroviruses from the family called Picornoviridae. It is most commonly caused by Coxsackie virus (A16), human enterovirus (HEV71) or other enteroviruses including Coxsackie virus A (CAV) 4, 5, 9 and 10 or Coxsackie virus B (CBV) 2 and $5[1,2,3]$.

Recent outbreak of HFMD in countries such as Taiwan, Western Australia and Singapore had brought the world attention to HFMD due to complications of death related cases. $[4,5]$ researched on HFMD in Taiwan and [3] did a phylogenetic analysis in Western Australia, Singapore and Malaysia on HEV71 while [6, 7] studied HFMD in Singapore. Most of the existing studies on HFMD are of clinical aspects.

Study done by [1] on HFMD in Sarawak revealed that Sarawak has been experiencing outbreak of
HFMD once every three years since 1997 and predicted that an outbreak would occur in 2006. The outbreak did occur in 2006 and health authorities in Sarawak confirmed this revelation as mentioned in [8, 9].

The outbreak in 2006 resulted in 13 deaths and 14,423 children infected. Besides that HFMD outbreak also caused social welfare and economic problems in Sarawak. In order to reduce the spread of HFMD, all child day-care centres, kindergarten and primary one to primary three classes were ordered to be closed for two weeks in $2006[10,11]$. The closure had caused problems for working parents who had to find alternative in caring for the children that were affected by the closure. Parents were requested to keep the children indoor and away from any crowded places such as supermarket and playgrounds.

It can be seen that HFMD not only caused health problems but also social and economical problems which are not easily quantifiable. So it is important to understand the spread of HFMD using mathematical model. Using the model we can predict the spread of HFMD in terms of number of infected and the duration of the outbreak. This will enable policy makers to take effective measures to curb the spread and reduce the effect of the disease.

In the following section we will describe the clinical characteristic of HFMD. This is followed by the formulation of the mathematical model that is going to be used to predict the spread of HFMD. In section four, we will present the numerical results that were obtained and finally we discuss a threshold value which is sometimes known as the basic reproduction ratio that controls the spread of the disease.

\section{Hand, foot and mouth disease (HFMD)}

Multiple outbreaks of HFMD were recorded around the world usually in summer for temperate countries 
and at any time during the year in tropics countries. In recent years, countries such as Taiwan, Singapore and Malaysia experienced HFMD outbreak that resulted in multiple deaths due to complications with HFMD. In Malaysia HFMD is an endemic that occurs every year in Sarawak especially from February to June. Sarawak has been experiencing the outbreak of HFMD in 1997, 2000, 2003 and 2006. The next predicted outbreak will be in the year 2009 [8]. The recorded number of person infected and the number of deaths for each outbreak is tabulated below.

Table 1. Number of cases and deaths related to HFMD outbreak in Sarawak [8],[9]

\begin{tabular}{|c|c|c|}
\hline Year & $\begin{array}{c}\text { Number of } \\
\text { cases }\end{array}$ & $\begin{array}{c}\text { Number of } \\
\text { deaths }\end{array}$ \\
\hline 1997 & 2626 & 31 \\
\hline 2000 & 3560 & 0 \\
\hline 2003 & 2113 & 0 \\
\hline 2006 & 14,423 & 13 \\
\hline
\end{tabular}

\subsection{Clinical characteristics}

The following clinical characteristics of HFMD are summarized from the multiple studies done by $[1,9$, 12] on HFMD. The warning issued by the health authority stated that the susceptible are the children below the age of ten years [9].

A person who is exposed to HFMD viruses will exhibit the symptoms after three to seven days. This is considered the incubation period of HFMD. Fever is usually the first symptoms of HFMD followed by poor appetite, malaise and sore throat. One or two days after the fever begins, small red spots develop in the mouth that blister and often develop into ulcers. These are mostly found on the tongue, gums and inside of the cheeks. The skin rash develops over one or two days with flat or raised red spots, some with blisters on the palms of the hand and the soles of the feet. Thus, the name of the disease is hand, foot and mouth disease (HFMD). A person with HFMD may some time have only the rash or the mouth ulcers.

HFMD is considered highly contagious among children. A person is most contagious during the first week of the illness. As the viruses are present in the throat and stools of an infected person, infection generally occurs via the faecal-oral or via contact with skin lesions and oral secretions. The virus may continue to be excreted in the stools of infected persons up till one month. The spread of the virus does not involve any vectors.
At the moment there is no specific antiviral drug to cure HFMD. There is also no vaccine available for the treatment of HFMD. Infected person is usually given medication to provide relieve from the pain caused by fever, aches or mouth ulcers. Victims are asked to take plenty of liquid. An infected person will fully recover after 7 to 10 days.

There is no permanent immunity against HFMD as the disease is caused by a group of viruses much like the case of flu. A person who recovered from the HFMD caused by Coxsackie A is susceptible to HFMD caused by enteroviruses 71 or any other enteroviruses.

\section{2}

\section{Related works}

[13] used the SIR model to analyze the association between the weather and the occurrence of enterovirus complicated severe cases in Taiwan. The model is based on transformation function of seasonal factors that determines the transmission coefficient. [14] attempted to establish nonlinear mathematical models in order to simulate the incremental effects of global warming on HFMD incidences in Tokyo. The two models tried to find the relationship between the outbreaks of HFMD with the weather patterns in the respective countries.

In Sarawak, a few studies had been done on HFMD. Most of them focussed on the clinical and pathological [12] together with the virological and molecular [15] aspect of HFMD. The studies did not reveal anything regarding the transmission coefficient and the immunity against HFMD. However, the study done by [12] revealed that the victims of HFMD were between the ages of 0.5 to 5.9 years with the mean of 1.7 years. Study done in [1] revealed that the victims were between the ages of 18 days to 155 months with the mean of 32.2 months. Even though the result may suggest that the susceptible group might only be children below the age of four or younger, we still maintain the susceptible group as children below the age of ten.

\section{Mathematical modelling}

Owing to the severity of HFMD outbreak in Sarawak, it is important to formulate a mathematical model to better understand the dynamics of the disease. These will enable a better decision making for the health policy makers. 